Jefferson A. Alamani, MD

Elias T. Reala, MD

Samantha S. Castaneda, MD

Antonio H. Chua, MD

Department of Otolaryngology

Head and Neck Surgery

Jose R. Reyes Memorial Medical Center
Correspondence: Dr. Jefferson A. Alamani

Department of Otolaryngology

Head and Neck Surgery

Jose R. Reyes Memorial Medical Cente

San Lazaro Compound, Rizal Avenue

Sta. Cruz, Manila 1003

Philippines

Phone: (632) 7436921

(632) 7119491 local 320

Email: dyeph.kk10@gmail.com

Reprints will not be available from the author.

The authors declared that this represents original material that is not being considered for publication or has not been published or accepted for publication elsewhere, in full or in part, in print or in electronic media; that the manuscript has been read and approved by all the authors, that the requirements for authorship have been met by each author and that each author believes that the manuscript represents honest work.

Disclosures: The authors signed disclosures that there are no financial or other (including personal) relationships, intellectual passion, political or religious beliefs, and institutional affiliations that might lead to a conflict of interest.

\section{Drain versus No Drain After Thyroidectomy: A Preliminary Prospective Randomized Controlled Trial}

\begin{abstract}
Objective: To evaluate the necessity of placing a drain in post-thyroidectomy patients, we aimed to determine whether insertion of a passive drain as compared to no drain in post-thyroidectomy patients would significantly affect hematoma formation, wound infection, wound dehiscence and length of hospital stay.
\end{abstract}

\section{Methods:}

Design: Prospective randomized controlled trial

Setting: Tertiary government training hospital

Subjects: Patients who underwent thyroidectomy for various thyroid pathologies were divided into two postoperative treatment arms: one group with insertion of a passive drain, and another group without a drain. Hematoma, wound infection, wound dehiscence and length of hospital stay were the outcomes measured per treatment arm.

Results: A total of 66 patients were evaluated. There were 54 females (81.81\%) and 12 males $(18.18 \%)$. The mean age for the drain group was 44.88 years and 43.67 years for the no drain group. Four patients developed complications in the drain group and two developed complications in the no drain group. The rate of complications between both groups was not statistically significant. The mean hospital stay of the drain group was 3.15 days which in the no drain group was 2.51 days. The difference in length of hospital stay was statistically significant.

Conclusion: There was no difference in the development of complications among the drain and no drain group. Thyroidectomy without surgical drains was associated with a significant reduction in hospital stay compared to thyroidectomy with routine placement of drains.

Keywords: surgical drainage / methods, hematoma / prevention and control, postoperative complications / prevention and control, thyroid disease / surgery, thyroidectomy / methods

Many surgeons practice the tradition of leaving a drain after thyroid surgery with the hope that this will obliterate dead space and prevent hematoma and seroma formation in the thyroid bed by passive evacuation. ${ }^{1,2,3,4}$ This belief is further reinforced by the fact that postoperative drains usually yield fluid. However, blood and serum that they are supposed to drain may block drains. Drains also add to discomfort, give extra scar and may increase hospital stay. ${ }^{1}$ Khanna et al. pointed out that placement of drains after routine thyroid surgery may induce rather than prevent 
fluid collection, is not related to the type of surgery or size of nodule, has no influence on complications, leads to an extra scar and may increase the hospital stay. ${ }^{2}$ They added that meticulous hemostasis and attention to finer details during surgery are more important. Routine use of drains after thyroid surgery may therefore not be necessary. Colak added that postoperative complications cannot be prevented by using drains after total thyroidectomy or lobectomy for benign thyroid disorders. ${ }^{1}$ Moreover, the use of drains may increase postoperative pain and the analgesic requirement and prolong the hospital stay. ${ }^{1}$ Tabaqchali observed 606 thyroid procedures and concluded that there was a significant increase in the rate of postoperative bleeding/ hematoma in patients with a drain. ${ }^{3}$ Wound infection occurred only in the patients with a drain. There was no difference in the incidence of postoperative bleeding and airway obstruction between the drain and selective groups. ${ }^{3}$ And yet, the widespread use of drains persists, prompting us to undertake this study.

Objective: To evaluate the necessity of placing a drain in postthyroidectomy patients, we aimed to determine whether insertion of a passive drain as compared to no drain in post-thyroidectomy patients, would significantly affect hematoma formation, wound infection, wound dehiscence and length of hospital stay.

\section{METHODS}

This was a prospective Randomized Controlled Trial conducted in a tertiary government training hospital in the Philippines from April 2012 to June 2013.

Participants: All patients seen at the outpatient department and diagnosed with a thyroid neoplasm were considered for inclusion. Diagnoses included nodular and multinodular non-toxic goiter with normal thyroid function tests and fine needle biopsy suggesting benign disease (nodular goiter, colloid goiter, hyperplastic / adenomatous nodule, Hashimoto's thyroiditis), as well as well-differentiated tumors (papillary thyroid carcinoma, follicular neoplasm). Surgical plans included total thyroidectomy, total thyroid lobectomy or completion thyroidectomy approved by a subspecialty consultant or with a consensus from a pre-operative conference. No patient was excluded on the basis of size of the gland, laterality or bi-laterality, difficulty of surgery, or re-operation in the neck. Following patients were excluded:

1. extensive malignancy such as skin metastasis

2. mediastinal extension

3. cervical lymph node metastases requiring neck dissection

4. clinical or laboratory indicators of coagulation disorders

5. No consent
The sample size was computed as follows:

\section{Sample size}

$$
\mathbf{n}=\frac{\underline{t}^{2} \times p(1-p)}{\mathbf{m}^{2}}
$$

Description:

$\mathbf{n}=$ required sample size

$\mathbf{t}=$ confidence level at $95 \%$ (standard value of 1.96)

$\mathbf{p}=$ estimated prevalence of complications of thyroid cases $-2 \%$

$\mathbf{m}=$ margin of error at $5 \%$ (standard value of 0.05 )

*In general, serious complications occur in fewer than $2 \%$ of all thyroid cases $^{5}$

$$
\begin{aligned}
\mathbf{n} & =1.96^{2} \times 0.02(1-0.02) / 0.05^{2} \\
& =3.8416 \times 0.0196 / .0025 \\
& =0.0752954 / .0025 \\
\mathbf{n} & =30.11816 \\
\mathbf{n} & =\mathbf{3 0} \text { per group, total number of patients for the study } \mathbf{6 0}
\end{aligned}
$$

\section{Pre-operative Preparation}

With approval from the hospital review board and ethics committee, all patients seen at the Ear Nose Throat Outpatient Department and Emergency Room fulfilling the inclusion and exclusion criteria for this study were given a short lecture on thyroid nodules and surgery and regarding the use of drains. All prospective participants underwent pre-operative evaluation that included thyroid function tests, thyroid ultrasound and fine needle biopsy. Written informed consent was obtained and each participant was admitted and prepared for the thyroid surgery.

All participants also underwent routine preoperative and postoperative video laryngoscopy to verify the pre-operative vocal fold status and movement, documenting that paralysis was not present. The patients were randomly allocated to drain and non-drain group on the basis of computer-generated random numbers (Microsoft Excel). The randomization was performed by an independent staff.

\section{Intra-operative Procedure}

Six surgeons with equal thyroid surgery competency were rotated to perform the surgeries using the standard techniques for total thyroidectomy, thyroid lobectomy and completion thyroidectomy with the use of monopolar electrocautery and silk suture ligatures.

Each surgeon was only informed of the randomized intervention (drain or no drain) just before the closure of the skin incision. In the drain group, a well-ventilated half-inch rubber drain was used. 


\section{Post-operative Evaluation}

A single researcher monitored the progress of each patient noting any complications that occurred. The rubber drains were removed after 48 hours. Outcomes measured were the following:

1. Bleeding: Complications included post-operative minor bleeding described as bruises or hematoma that did not require re-operation and major bleeding which required reopening of the surgical field.

2. Wound infection: wound or surgical site infection (SSI) was determined in accordance with Centers for Disease Control and Prevention (CDC) guidelines by Horan et al. ${ }^{6}$

a. Infection occurs within 30 days of procedure

b. Involves only skin or subcutaneous tissue around the incision.

c. And at least one of the following:

i. Purulent drainage from superficial incision

ii. Organism isolated from aseptically obtained culture of fluid or tissue from superficial incision

iii. At least one of the following signs and symptoms of infection: pain or tenderness, localized swelling, redness or heat and superficial incision is deliberately opened by surgeon, unless culture of incision is negative.

iv. Diagnosis of superficial incisional SSI by the surgeon or attending physician.

3. Wound dehiscence: persistent open surgical wound observed at one week post- operatively

4. Length of hospital stay: days recorded from the day of surgery to the day of discharge

All specimens were subjected to histopathological examination for final confirmation of diagnosis. Data were encoded and tallied in Microsoft Excel. Descriptive statistics were generated for all the variables. Nominal data, frequencies and percentages were computed. For numerical data, means with standard deviation (SD) were computed. Analysis of the different variables was done using T-test and Fisher Exact test.

\section{RESULTS}

A total of 66 patients [12 males (18.18\%), 54 females (81.81\%), mean age 44.25 years, range 20-80] fulfilled the inclusion and exclusion criteria. The drain group served as the control group and the no drain group served as the treatment group. Thirty-two patients ( 6 males, 26 females) were in the drain group with 34 patients ( 6 males, 28 females) in the no drain group.

There was no statistically significant difference in demographic characteristics of age and gender between the two groups ( $p$ value $<$ 0.05). (Table 1) The patients were older in the drain group (mean age 44.73 years, range of 21- 80) than the no drain group (mean age 43.79 years, range 20-66) but the mean age difference between groups was not statistically significant. (Table 1)
Of the 32 patients in the drain group, 20 underwent total thyroidectomy and 12 underwent lobectomy. In the no drain group, 9 underwent total thyroidectomy and 25 underwent lobectomy. This comparison of operations performed in both groups was statistically significant $(P$ value $=0.006)$.

Most of the specimens $(62 / 66$ or $93.94 \%)$ were forwarded to the Pathology Department of the hospital, submerged in formalin solution (potentially resulting in smaller measurements) and were measured by an independent pathology resident. In the drain group, the mean specimen volume was $132.61 \mathrm{~cm}^{3}$ (range $15.36-554.5 \mathrm{~cm}^{3}$ ) while the no drain group specimen volume was $73.06 \mathrm{~cm}^{3}$ (range of 12.0-357.5 $\mathrm{cm}^{3}$ ). The difference in specimen volume between groups was not statistically significant $(\mathrm{P}$ value $=0.06)$. (Table 1$)$

The final histopathologic reports in the drain group were 12 welldifferentiated thyroid carcinoma and 20 benign compared to 8 welldifferentiated thyroid carcinoma and 26 benign in the no drain group. This distribution was not statistically significant $(P$ value $=0.29)$. (Table 1)

The mean operative time for the procedure from skin incision to closure was 243.19 minutes (SD 66.57) in the drain group and 209.24 minutes (SD 79.68) in the no drain group. The difference in operative time between the two groups was statistically significant at $p$ value of 0.02 (Table I). This difference could be due to the number of total thyroidectomies done in the drain group compared to the no drain group translating into longer operative time.

The amount of blood loss in milliliters was also recorded, an estimation of the blood suctioned and operating sponges consumed which the anesthesiologist noted. The drain group had a mean of 318.79 $\mathrm{ml}$ (SD 252.91) versus $262.42 \mathrm{ml}$ (SD 158.69) in the no drain group. The difference of blood loss was not statistically significant at $p$ value of 0.13 (Table 1).

The two groups were relatively equal in terms of age, gender distribution, blood loss and final histopathological report. There were significant differences in operation performed and operative time. (Table 1) The increase number in the lobectomy operation compared to total thyroidectomy translated to the increased duration of operation.

There were a total of six complications out of 66 patients recorded during the study, a rate of $9.09 \%$. Of the 32 patients in the drain group, four developed complications, while two complications were observed out of 34 patients in the no drain group. (Table 2) The four complications of the drain group included two bleeding which were both expanding hematomas that needed re-opening for ligation of bleeders and evacuation and two wound dehiscences. Both complications from the no drain group were wound dehiscences. No patient developed wound infection. The comparison of occurrence of complications between the two groups was not statistically significant $(P$ value $=0.10$ ) 


\section{ORIGINAL ARTICLES}

Table 1. Comparison of Demographic Characteristics between the Two Groups

\begin{tabular}{|c|c|c|c|}
\hline & $\begin{array}{l}\text { Drain } \\
(n=32)\end{array}$ & $\begin{array}{c}\text { No Drain } \\
(n=34)\end{array}$ & P value \\
\hline$\frac{\text { Age in years }}{\text { Mean } \pm S D}$ & $44.73 \pm 14.30$ & $43.79 \pm 12.96$ & 0.36 (NS) $\dagger$ \\
\hline $\begin{array}{c}\frac{\text { Gender }}{\text { Female }} \\
\text { Male }\end{array}$ & $\begin{array}{c}26(81.25 \%) \\
6(18.75 \%) \\
\end{array}$ & $\begin{array}{c}28(82.53 \%) \\
6(17.64 \%) \\
\end{array}$ & 1.0 (NS) * \\
\hline$\frac{\text { Volume of Thyroid in }}{\frac{\text { cubic centimeters }}{\text { Mean } \pm \text { SD }}}$ & $132.61 \pm 176.85$ & $73.06 \pm 75.28$ & 0.06 (NS) $\dagger$ \\
\hline $\begin{array}{c}\text { Operation Performed } \\
\text { Total Thyroidectomy } \\
\text { Lobectomy }\end{array}$ & $\begin{array}{l}20 \\
12\end{array}$ & $\begin{array}{c}9 \\
25\end{array}$ & $0.006(S)^{*}$ \\
\hline$\frac{\text { Blood loss in }}{\frac{\text { Millimeter }}{\text { Mean } \pm \text { SD }}}$ & $318.79 \pm 252.91$ & $262.42 \pm 158.69$ & 0.13 (NS) $\dagger$ \\
\hline $\begin{array}{c}\frac{\text { Operative time in }}{\text { minutes }} \\
\text { Mean } \pm \text { SD }\end{array}$ & $243.19 \pm 66.57$ & $209.24 \pm 79.68$ & $0.02(S) \dagger$ \\
\hline $\begin{array}{c}\text { Histopathological } \\
\text { Report } \\
\text { Benign WDTC }\end{array}$ & $\begin{array}{l}20 \\
12\end{array}$ & $\begin{array}{c}26 \\
8\end{array}$ & 0.29 (NS) * \\
\hline
\end{tabular}

Table 2. Rate of Post-operative Complications

\begin{tabular}{|c|c|c|c|}
\hline Complications & $\begin{array}{c}\text { Drain } \\
(\mathbf{n}=\mathbf{3 2})\end{array}$ & $\begin{array}{c}\text { No Drain } \\
(\mathbf{n}=\mathbf{3 4})\end{array}$ & \\
\hline Bleeding & $2(6.25 \%)$ & 0 & $\mathbf{0}$ \\
\hline Wound Infection & 0 & 0 & $\mathbf{0}$ \\
\hline Wound Dehiscence & $2(6.25 \%)$ & $2(5.88 \%)$ & $\mathbf{0}$ \\
\hline Total & $\mathbf{4 ( 1 2 . 5 \% )}$ & $\mathbf{2 ( 5 . 8 8 \% )}$ & P value $\mathbf{0 . 1 0}$ (NS) \\
\hline
\end{tabular}

Legend: $\mathrm{SD}=$ Standard Deviation, NS = Not Significant

The comparison of length of hospital stay (surgery to discharge) between the two groups showed a significant difference at $P$ value of $<0.05$. The distribution of length of stay is shown in Figure 1. The mean length of hospital stay in the drain group was 3.15 days (range 2 to 6 days, SD 0.99). The mean length of hospital stay in the no drain group was 2.51 days (range 2 to 6 days, SD 0.89). The mean difference in length of hospital stay between the two groups was statistically significant $(P$ value $=0.003$ )

†T-test *Fisher Exact test

Legend: $\mathrm{SD}=$ Standard Deviation, NS $=$ Not Significant

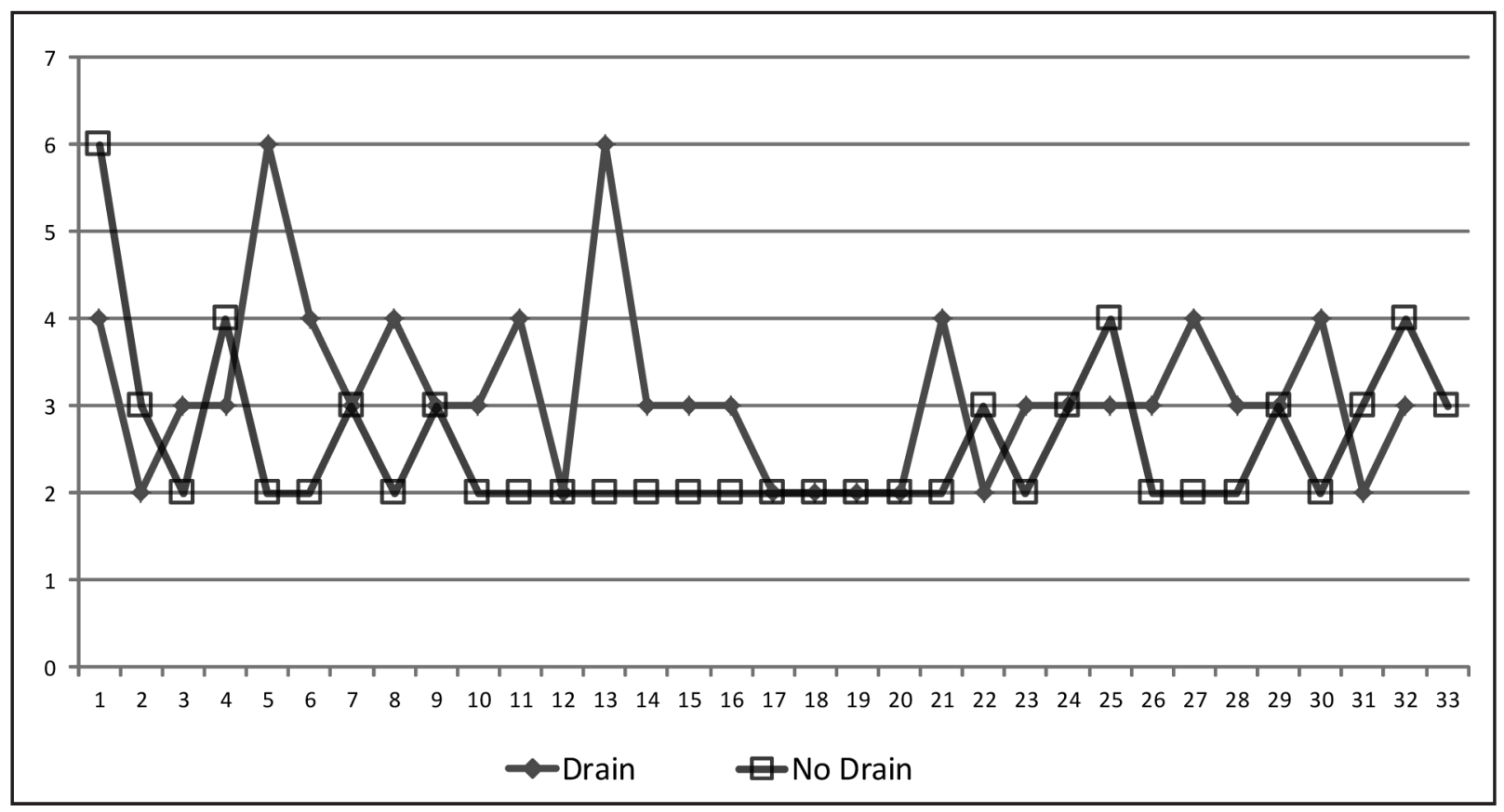

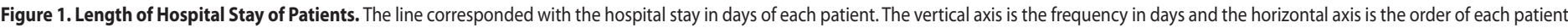
randomly allocated on each group. The Drain group was plotted with rhombus while the No Drain was plotted with squares. 


\section{DISCUSSION}

Are surgical wound drains used by head and neck surgeons after thyroidectomy really needed? We evaluated the necessity of placing a drain post-thyroidectomy and compared the difference in complication rates and hospital stay between the drain and no drain groups.

The difference in complication rates between the two groups was not statistically significant. Complications occurred in both groups. All complications that occurred in the two groups ( 4 in the drain group and 2 in the no drain group) were in-patients with a final histopathologic result of well-differentiated thyroid carcinoma (papillary thyroid or follicular carcinoma). Could the complications of bleeding and wound dehiscence be related to angiogenesis promoted by malignancy and a higher probability of bleeding during thyroidectomy? Nevertheless, our findings suggest that postoperative complications cannot be prevented by drains after thyroid surgery, in consonance with the literature. ${ }^{1-4}$ The use of drains may not be necessary in most cases of thyroidectomy. Meticulous dissection and hemostasis remain the key to minimizing drastic complications in thyroid surgeries.

The occurrence of wound dehiscence in both groups may be attributed to seroma formation. Seroma is caused by division of lymphatic and adipose tissues during neck dissection. Seroma is defined as the collection of serous fluid in the potential space between cervical skin flaps and underlying structures. Seromas present as enlarging masses under the skin with or without overlying induration or erythema. ${ }^{5}$ A study by Shan et al. explained that the presence of seroma can lead to flap detachment, incision dehiscence and wound infection. ${ }^{7}$ The seroma that developed in four patients could have been due to increased subcutaneous dissection during elevation of skin flaps.

The difference in length of hospital stay between the two groups was statistically significant. The duration of stay was longer in the drain group compared to the no drain group. The placement of drain translated to longer days in the hospital as observed in the literature. ${ }^{1-4}$

In conclusion there was no difference in the development of complications among the drain and no drain group. Thyroidectomy without drains led to a significant reduction of hospital stay compared to the routine placement of drains.

\section{REFERENCES}

1. Colak T, Akca T, Turkmenoglu O, Canbaz H, Ustunsoy B, Kanik A, et al. Drainage after total thyroidectomy or lobectomy for benign thyroidal disorders; Univ Sci B 2008; 9(4):319-323.

2. Khanna J, Chintamani RSM, Bhatnagar D, Mittal MK, Sahoo M, Mehrota M. Is the routine drainage after surgery for thyroid necessary? - A prospective randomized clinical study, $B M C$ Surgery 2005;5:11

3. Tabaqchali MA, Hanson JM, Proud G. Drains for thyroidectomy/parathyroidectomy: fact or fiction?; Ann R Coll Surg Engl 1999; 81(5): 302-305.

4. Chalya Pl, Gilyoma JM, Mchembe M. Drain versus no drain after thyroidectomy: A prospective randomized clinical study. East Cent Afr J Surg. 2011; 16(2): 55-61.

5. Lai SY, Mandel SJ, Weber RS, Management of Thyroid Neoplasm in Flint PW, Haughey BH, Lund VJ, Niparko JK, Richardson MA, Robbins KT, Thomas JR. Cummings Otolaryngology Head and Neck Surgery, $5^{\text {th }}$ edition. 124:1750-72. Mosby Elsevier 2010.

6. Horan TC, Gaynes RP, Martone WJ, Jarvis WR, Emori TG. CDC definitions of nosocomial surgical site infections. A modification of CDC definitions of surgical wound infections. Infect Control Hosp Epidemiol 1992; 13 (10): 606-08.

7. Shan CK, Zhang W, Jiang XM, Liu S, Qui M. Prevalence, risk factors, and management of seroma formation after breast approach endoscopic thyroidectomy, World J Surg 2010 Aug; 34(8): 1817 22. 